\title{
MITIGATION OF INTERFERENCE IN UPLINK SPECTRUM BY SHIFTING HANDOVER BOUNDARY IN LTE HETEROGENEOUS NETWORKS
}

\author{
Mohammad T. Kawser ${ }^{1 *}$ and Nafiz Imtiaz Bin Hamid ${ }^{2}$ \\ ${ }^{1,2}$ Electrical and Electronic Engineering Department, Islamic University of \\ Technology, Bangladesh \\ 1'mkawser@hotmail.com, ${ }^{2}$ nimtiaz@iut-dhaka.edu
}

\begin{abstract}
A heterogeneous network (HetNet), typically a combination of macrocells and small cells, is a popular solution for Long Term Evolution (LTE) based 4G or 5G technologies as it can provide ubiquitous and cost-effective broadband user experience with higher network capacity. However, the interference issues are more complicated in LTE HetNets. The mobile and unknown locations of the User Equipment (UEs) add further difficulties to interference management in uplink spectrum where this uplink spectrum is a separate range from downlink for LTE FDD and the same range but on specific time slots for LTE TDD. Since the major source of this interference is the high power transmission from UEs, located in neighboring cells but far away from their serving eNodeBs, limiting this power can be greatly helpful. To mitigate interference in uplink spectrum by reducing power transmission from those UEs, a scheme is proposed in this paper for HetNets. The proposed scheme shifts the handover boundary from its conventional location for UEs, performing data transfer only in uplink. It can additionally help increase the battery life for these UEs and also, it limits a drop in uplink data rate due to clipping off power transmission level by the UE capability. Simulation has been performed to demonstrate the reduction in uplink power transmission in the proposed scheme.
\end{abstract}

Keywords - LTE, HetNets, Interference, Cell Selection Offset (CSO), Handover

\section{INTRODUCTION}

Modern cellular communication has reached its current pinnacle through LTE, along with its later versions LTE-Advanced, LTE-Advanced Pro, and 5G. In order to ensure greater enhancement of coverage and capacity, HetNets have been introduced in LTEAdvanced. Usually, regular macrocells, overlaid by low power small cells, namely, microcell, picocell, femtocell, remote radio head (RRH), and relay node (RN) constitute a HetNet [1]. In this paper, microcell, picocells, and femtocells are referred to as small cells and HetNets are assumed to be formed through them in combination with macrocells. In HetNets, the small cells complement the macro networks by improving coverage, adding targeted capacity and supporting new services and user experiences. The increased capacity from small cells can be especially helpful in densely populated urban areas. The small cells can also be used to fill in dead spots or to expand the coverage by adding them along the coverage rim. The operators are gradually adopting small cell deployment as an essential part of their strategy to meet future demand, resulting in a big industry push towards HetNets. However, many cell boundaries are formed in HetNets with different capabilities of eNodeBs, resulting in a very complicated interference problem. These interferences can

Received: May 6, 2018

Reviewed: August 12, 2019

Accepted: August 31, 2019

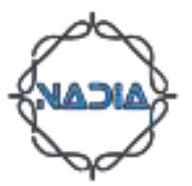


appear in various ways as explained by the authors of [2]. Signal-to-interference-plus-noise ratio (SINR) is degraded by a high interference that leads to the use of lower modulation and coding scheme (MCS) along with an increase in retransmissions of erroneous packets culminating in lower data rate.

In the case of frequency division duplex (FDD) version of LTE, paired but separate frequency ranges are used for downlink and uplink operations. On the other hand, in the case of time division duplex (TDD) version of LTE, the same frequency range is used and an uplink-downlink configuration defines how the time slots are allocated for downlink and uplink. The neighboring cells usually operate with the same uplink-downlink configuration to avoid UL-DL interference, especially, to ensure that the high transmit power of the eNodeB does not affect the transmission of a UE in a neighboring cell [3]. Single carrier frequency division multiple access (SC-FDMA) technique is used in LTE uplink. LTE allocates a user a few contiguous resource blocks (RBs), each having a width of $180 \mathrm{kHz}$. Thus, every user transmits on a narrow bandwidth and can cause interference to the reception of signal at eNodeB, from other users, transmitting on the same bandwidth. The interference issue is critical because most LTE deployments have 1:1 frequency re-use. Also, the interference management can often be more problematic in the uplink spectrum, compared to the downlink, as the sources of the uplink interference in the system are UEs, which have scattered, mobile, unknown and unpredictable locations in the cellular system. The uplink interference is managed in LTE through careful power control, which has undergone extensive research. To manage uplink interference in LTE, a measurement data driven machine learning paradigm for power control is proposed in [4]. The data driven approach has the inherent advantage that the solution adapts based on network traffic, propagation and network topology. In [5], for LTE HetNets, a low-complexity, distributed interference mitigation method is proposed. The method is expected to be aware of network load and propagation conditions. With universal frequency reuse, interference mitigation for uplink interference in HetNet is investigated in [6]. It proposes a user-specific resource allocation as well as a power control scheme by grouping the mobile users into two different groups as per their evaluated signal-to-leakage-plus-noise ratio (SLNR) metric. However, the outcome of the present research is limited primarily due to the fact that the UEs, which are located far away from the eNodeB, use high transmit power and cause interference in neighboring cells in uplink spectrum. Thus, limiting this power transmission could rein in the generation of interference in the system.

Depending on the type of application, the data transfer for the user can be downlink only, uplink only or bidirectional, for example, in the case of FTP upload, FTP download, and video call, respectively. In HetNets, in the case of downlink signal, the maximum transmit power capability is a lot different between the macrocell and the small cell. However, this is not the case for uplink, since the UEs, wherever they are located, have ideally the same maximum transmit power capability. Thus, the handover boundary, which depends on the transmit power level, appears to have different requirements between downlink and uplink operations. It may be noted that this difference in requirement does not exist in homogenous networks, which have equal transmit power for all eNodeBs [7]. However, at present, it is always the downlink signal, received at the UE, which is used to make a handover decision. So, the same handover boundaries are used regardless of the direction of data transfer. This does not seem warranted in HetNets for applications with uplink only data transfer. When a UE moves from small cell to macrocell, it suddenly increases the uplink transmit power substantially as the handover is triggered due to a large distance from the macro eNodeB. Similarly, when a UE moves from macrocell to small cell, it transmits high uplink power to macrocell recently before the handover is triggered. The interference, caused to the neighboring cells, by the use of this high uplink power transmission, is significantly high because the LTE system typically uses 1:1 frequency re-use. In this paper, a scheme is proposed to mitigate this interference by shifting handover boundary HetNets and this 
measure can be applied only to the UEs, which are performing data transfer only in uplink. The proposal also saves battery power for these UEs.

The rest of this paper is arranged as follows. Cell range expansion (CRE) to bias handover condition is investigated in Section 2. Robustness of downlink control information, supporting uplink only data transfer, is covered in Section 3. In Section 4, we present the proposed scheme that shifts the handover boundary in HetNets for UEs performing data transfer only in uplink. Simulation results that exhibit the achievements of the proposed scheme are shown in Section 5 and through Section 6, the whole paper comes to conclusion.

\section{CRE TO BIAS HANDOVER CONDITION}

The eNodeB compares the radio link quality from the serving and neighboring cells, as received by the UE and issues a handover command when the neighboring cell stays better than the serving cell by an adequate margin persistently for a sufficient period. For this purpose, the serving eNodeB receives the information of the downlink signal strength of different cells via measurement reports from the UE. Usually, after receiving a certain number of measurement reports, with the radio signal better from the target cell compared to the serving cell by a margin, the handover command can be issued. Measurement reporting has two types, namely, event-triggered reporting and periodic reporting as shown next [1], [9].

- Event-Triggered: When certain conditions are met, the UE enters and leaves a particular event. To enter the event, the conditions need to be fulfilled for TimeToTrigger period. While the event remains activated, the UE sends measurement reports, one after another, at certain time intervals, until the maximum permissible number of reports is reached. For intra-frequency handover, five types of events are defined, known as A1, A2, A3, A4, and A5. The condition to enter event A3, the most widely used event, is

Neighbor cell measurement + OffsetFreq for neighbor cell + Cellindividualoffset for neighbor cell - Hysteresis $>$ Serving cell measurement + OffsetFreq for serving cell + CellindividualOffset for serving cell + A3_Offset

- Periodical: The measurement reports are continuously sent at certain time intervals until the maximum number of reports is reached.

In HetNets, the macro eNodeB transmits much higher power compared to the eNodeB of small cell. Therefore, many UEs, closer to the eNodeB of small cell, can be served by the macrocell, resulting in unbalanced load distribution among tiers. To remedy this problem, the cell range expansion (CRE) can be used, which favors handover decision towards the small cell [8]. For this purpose, a virtual cell selection offset (CSO) is used in (1) to bias toward the small cells and expand its range. The eNodeB sends the fields 'CellIndividualOffset for serving cell' and 'CellIndividualOffset for neighbor cell' on the RRCConnectionReconfiguration message. The CellIndividualOffset field values are set by the field Q-OffsetRange, which can range between $-24 \mathrm{~dB}$ and $24 \mathrm{~dB}$ [9]. The CellIndividualOffset fields can be used to set CSO.

\section{ROBUSTNESS OF DOWNLINK CONTROL INFORMATION SUPPORTING UPLINK ONLY DATA TRANSFER}

When an application runs with data transfer, only in uplink directions, the UE receives and decodes downlink reference signals and a few downlink control channels. The 
downlink reference signals are primarily used for channel estimation. The robustness of the downlink control channels against poor channel quality is explained below [1].

- Physical Control Format Indicator Channel (PCFICH): PCFICH indicates whether the number of OFDM symbols for control region in the subframe is 1,2 or 3. PCFICH uses QPSK and $(32,2)$ block code resulting in $1 / 16$ coding rate. Thus, PCFICH is highly robust.

- Physical Downlink Control Channel (PDCCH): DCI format 0 of PDCCH is used to convey the uplink resource grants for the UE. It carries resource block assignment and hopping information, MCS, new data indicator (NDI) field, and so forth. PDCCH uses QPSK and tail biting convolutional coding (TBCC) leading to significantly high robustness.

- Physical Hybrid ARQ Indicator Channel (PHICH): PHICH carries the HARQ ACK/NACK indicating if an uplink transport block has been correctly received or not. Multiple PHICH instances are code multiplexed and separated through different orthogonal sequences in a PHICH group. The maximum number of $\mathrm{PHICH}$ instances in a PHICH group is eight for normal cyclic prefix and four for extended cyclic prefix. A PHICH group is BPSK modulated and mapped on 3 REGs or 12 resource elements leading to pretty high robustness. PHICH is typically transmitted only on the first symbol of the subframe. However, if the radio link is very poor then PHICH is extended to more number of symbols to increase robustness. Thus, PHICH also possesses significantly high robustness.

It is thus evident that the downlink control channels, supporting an uplink only data transfer, are highly robust against poor channel quality.

\section{PROPOSED SCHEME}

It is assumed that there are UEs in the cells, which are performing data transfer only in uplink. It is also assumed that LTE uses either FDD version or TDD version with the same uplink-downlink configuration in the neighborhood. Thus, the uplink transmission uses the same spectrum at any time instant in a neighborhood. The interference issue, in general, can be very critical in HetNets [2]. The interference in uplink spectrum in a cell is experienced primarily because of the power transmission of UEs in the neighboring cells, and this interference can be significantly high with the typical use of 1:1 frequency re-use. However, the uplink power increases as the UE moves further away from the serving eNodeB. So, in the area, near the conventional handover boundary, the UEs use transmit power higher than other areas of the cell. Since this area is away from the serving eNodeB, it is close to the neighboring eNodeBs. As the UEs radiate equally in all horizontal directions, in the existing scheme, the UEs, in that area, are the major sources of interference to the reception of signal at neighboring eNodeBs in HetNets. Therefore, limiting the power transmission of UEs, near the conventional handover boundary, can be an effective measure to mitigate interference in uplink spectrum in the system. A scheme is proposed in this section, which limits the power transmission as such but only for the UEs, which are performing data transfer only in uplink. The proposed scheme shifts the handover boundary from its conventional location only for those UEs.

Since the handover decision is always based on the downlink signal received at the UE, and the macrocell transmits much higher power than the small cell does, the position of triggering handover is closer to small cell, in the existing scheme, whether the UE moves from macrocell to small cell, or from small cell to macrocell. Thus, as explained in Section 1, the conventional moment of triggering handover leads to uplink interference in LTE HetNets. In this section, we propose setting CSO such as to shift the handover position 
roughly to the center between the eNodeBs of macrocell and small cell, if there is data transfer only in uplink. In the proposed scheme, when the UE moves from macrocell to small cell and undergoes data transfer only in uplink, its sudden increase in uplink power transmission and the associated interference will be mitigated by triggering the handover earlier than normal. Similarly, when the UE moves from small cell to macrocell and undergoes data transfer only in uplink, the handover will be triggered later than normal to preclude high uplink power transmission and its associated interference. Thus, different handover boundaries will be used between the cases when there is data transfer only in uplink and when there is any downlink only data transfer or bidirectional data transfer. For the purpose of necessary adjustment of CSO, in order to shift the handover boundary roughly to the middle between the two eNodeBs of macrocell and small cell, the CellIndividualOffset field on RRCConnectionReconfiguration message will be used, as explained in Section 2.

The proposed middle position for handover minimizes the maximum distance of the UE from both the eNodeBs of macrocell and small cell at the moment of triggering handover. So, the proposed scheme reduces the uplink transmit power for UEs, performing data transfer only in uplink, when the UEs are located near the conventional handover boundary. In this area, the uplink power approaches its maximum, and also, the UE may often be approaching the occurrence of a handover. Thus, it also minimizes the maximum uplink transmit power level used. The benefits of this proposed scheme are summarized below.

i. Mitigation of Uplink Interference: The proposed scheme deters some UEs from high power transmission and these UE are performing data transfer only in uplink and located in the area of the cell where high transmission power is otherwise used. Thus, the proposed scheme can substantially mitigate the overall uplink interference in the system for LTE HetNets. This is the key benefit of the proposed scheme.

ii. Longer Battery Life: As the UEs, performing data transfer only in uplink, use lower uplink transmit power within particular locations in the cell and so, their batteries will drain out less and the battery life will be longer.

iii. Mitigation of the Drop in Uplink Data Rate: The maximum power capability of the UE depends on its power class. The power received at the eNodeB can be lower than expected if the uplink transmit power gets limited or clipped off by the maximum power capability of the UE. Consequently, the data rate in uplink will also be less. As the proposed scheme lowers the uplink transmit power requirement when the uplink power is approaching its maximum, there will be slimmer chances of getting the uplink transmit power clipped off by the maximum power capability. In case, the clipping off occurs, both the amount of clipping and the period, during which clipping prevails, will be lower compared to the existing scheme. Thus, the associated drop in uplink data rate will also be lower in the proposed scheme. This benefit also applies only to the UEs, performing data transfer only in uplink.

The macrocell has wider coverage than small cell. So, in the existing scheme, a UE connected to the macro eNodeB goes further away from the eNodeB without any handover, compared to what a UE, served by the small cell, does. Thus, the maximum uplink transmit power is less in the latter case. Consequently, the mitigation of the uplink interference as well as two other aforementioned benefits, in the proposed scheme, will be more pronounced when the UE moves from macrocell to small cell, compared to when the UE moves from small cell to macrocell.

There can be a misconception that the necessary decoding of downlink channels, supporting data transfer in uplink, may fail in the proposed scheme. This apparent shortcoming of the proposed scheme is based on that there can be fairly lower received power in downlink from the serving eNodeB of small cell, when the UE is located in the area of the cell where the benefit of reduced uplink power is attained. This area can be 
designated as the place after the handover when the UE moves from macrocell to small cell and the place before the handover when the UE moves from small cell to macrocell. However, in practice, the lower downlink power reception can be expected to allow correct decoding. This is because, as explained in Section 3, the downlink control channels, supporting uplink only data transfer, can operate with high robustness against poor channel quality. Also, apart from the control channels, the physical downlink shared channel (PDSCH) may have to carry information in downlink, for example, TCP acknowledgments, during uplink only data transfer. But these transmissions on PDSCH occur infrequently and more importantly, using a low MCS level, PDSCH can also be made significantly robust.

\section{SIMULATION}

Simulation has been performed to demonstrate the reduction of the use of uplink transmit power in the proposed scheme, for UEs with uplink only data transfer. The simulation is MATLAB based and the simulation assumptions are shown in Table I, in which the assumed parameter values comply with 3GPP specifications [9]. It is assumed here that a user moves from macrocell to small cell and vice versa in a straight path connecting the eNodeBs of macrocell and small cell, as shown in the trajectory of Figure 1.

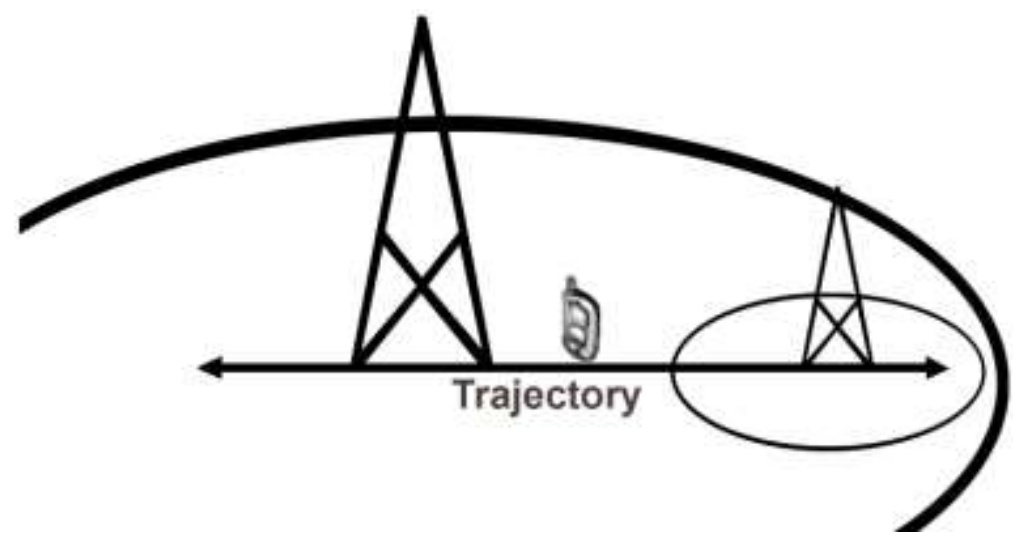

Fig. 1 The Trajectory of UE Movement

According to (1), when the UE moves from macrocell to small cell, the condition to begin sending measurement reports can be shown as

$$
\operatorname{Pr}(\text { small })-\text { Hysteresis }>\operatorname{Pr}(\text { macro })+A 3_{\text {offset }}
$$

where $\operatorname{Pr}($ macro $)$ and $\operatorname{Pr}($ small $)$ are the downlink power received at the UE from the macrocell and small cell, respectively. They can be calculated using

$\operatorname{Pr}($ macro $)=E I R P($ macro $)+\operatorname{Gr}(d B)-P L($ macro $)$

and

$\operatorname{Pr}($ small $)=E I R P($ small $)+G r(d B)-P L($ small $)$

where $G r$ is the antenna gain of the UE and PL(macro) and $P L($ small) are the downlink path loss in macrocell and small cell, respectively. The path loss models, shown in Table I, are used to calculate $P L($ macro $)$ and $P L($ small).

For the existing scheme, the simulation results show that the condition of (2) is fulfilled at a distance $d=699 \mathrm{~m}$ from macro eNodeB. Assuming that the handover is triggered after $n$ number of measurement reports, the actual handover is triggered at the distance 
$d^{\prime}=d+(v \times T T T)+(n \times$ ReportInterval $\times v)$

where TTT represents TimeToTrigger. From simulation, the value of $d^{\prime}$ becomes $701 \mathrm{~m}$.

Table I. Simulation Assumptions

\begin{tabular}{|c|c|}
\hline Parameter & Value \\
\hline Separation between macro eNB and small eNB & $800 \mathrm{~m}$ \\
\hline User velocity, $v$ & $10 \mathrm{~km} / \mathrm{hr}(=0.00083 \mathrm{~km} / \mathrm{sec})$ Pedestrian speed \\
\hline User direction & $\begin{array}{l}\text { From macrocell to small cell and from small cell } \\
\text { to macrocell }\end{array}$ \\
\hline Path loss (Macro) in $\mathrm{dB}$ & $P L($ macro $)=128.1+37.6 \times \log _{10} d(\mathrm{~km})$ \\
\hline Path loss (Small) in dB & $\begin{array}{c}P L(\text { small })=-39+67 \times \log _{10} d(m) ; \mathrm{d}> \\
45 \mathrm{~m}\end{array}$ \\
\hline EIRP at macro eNB & $40 \mathrm{dBm}$ \\
\hline EIRP at small eNB & $23 \mathrm{dBm}$ \\
\hline Antenna gain of UE, $G r$ & $-1 \mathrm{~dB}$ \\
\hline Uplink transmission bandwidth & $1.8 \mathrm{MHz}$ \\
\hline Measurement Reporting method & Event-triggered with event $\mathrm{A} 3$ \\
\hline OffsetFreq for serving cell & $0 \mathrm{~dB}$ \\
\hline OffsetFreq for neighbor cell & $0 \mathrm{~dB}$ \\
\hline $\begin{array}{c}\text { CellIndividualOffset for serving cell (existing } \\
\text { scheme) }\end{array}$ & $0 \mathrm{~dB}$ \\
\hline $\begin{array}{c}\text { CellIndividualOffset for neighbor cell (existing } \\
\text { scheme) }\end{array}$ & $0 \mathrm{~dB}$ \\
\hline A3_Offset & $2 \mathrm{~dB}$ (IE value, 4 ) \\
\hline Hysteresis & $8 \mathrm{~dB}$ (IE value, 16 ) \\
\hline ReportInterval & $0.24 \mathrm{sec}$ \\
\hline No. of reports triggering $\mathrm{HO}, n$ & 4 \\
\hline TimeToTrigger & $1.28 \mathrm{sec}$ \\
\hline $\mathrm{P}_{\mathrm{O} \_\mathrm{PUSCH}}$ (macro) & $-92 \mathrm{dBm}$ \\
\hline $\mathrm{P}_{\mathrm{O} \_ \text {PUSCH }}$ (small) & $-110 \mathrm{dBm}$ \\
\hline$\alpha$ & 0.8 \\
\hline No. of RBs, MPUSCH & 10 \\
\hline$\Delta_{\mathrm{TF}}$ & 0 \\
\hline $\mathrm{f}_{\mathrm{TPC}}$ & 0 \\
\hline
\end{tabular}

According to the proposed scheme, CSO is applied in an attempt to cause the handover earlier, in the middle between macro and small cells. Since $d^{\prime} \approx d$ and the distance between the eNodeBs of macrocell and small cell is $800 \mathrm{~m}, \mathrm{CSO}$ can be set such that it makes $d=400 \mathrm{~m}$. To find out this required CSO value, $\mathrm{CSO}_{m t o s}$, (2) can be modified as

$$
\operatorname{Pr}(\text { macro })+A 3_{\text {offset }}=\operatorname{Pr}(\text { small })-\text { Hysteresis }+\mathrm{CSO}_{\text {mtos }} .
$$

In (6), parameter values are used from Table I, and $\operatorname{Pr}($ macro) and $\operatorname{Pr}($ small) are determined using (3) and (4) for $d=400 \mathrm{~m}$, which gives the value of $C S_{m t o s}$ equal to 49 $\mathrm{dB}$. The fields 'CellIndividualOffset for serving cell' and 'CellIndividualOffset for neighbor cell' are used to set $\mathrm{CSO}_{\text {mtos }}$. However, as shown in Section 2, the CellIndividualOffset values can provide $48 \mathrm{~dB}$ for CSO at the maximum. It shows that, with the proposed scheme, the permissible range for CellIndividualOffset needs to be increased.

The UE computes its transmit power as [1]

$P_{U}=P_{O_{-} P U S C H}+\alpha \times P L+10 \log _{10} M_{P U S C H}+\Delta_{T F}+f_{T P C}$

where $\mathrm{P}_{\mathrm{O} \_\mathrm{PUSCH}}$ is the basic parameter for power control and it is configured by the eNodeB. The UE estimates the path loss in downlink by measuring the linear average over the power 
contributions of the resource elements on cell specific reference signals within the considered frequency bandwidth and using the parameter, ReferenceSignalPower sent by the eNodeB. $\alpha$ allows the eNodeB to configure the degree to which the UE's estimate of the path loss in downlink would be taken into account in uplink power control. $\mathrm{M}_{\mathrm{PUSCH}}$ indicates the number of resource blocks (RBs) allocated for uplink transmission and it attempts to keep transmit power spectral density (PSD) constant. $\Delta_{\mathrm{TF}}$ allows the transmitted power per resource block to be adapted according to the level of MCS, and this compensation for MCS can optionally be deactivated, which sets $\Delta_{\mathrm{TF}}=0 . \mathrm{f}_{\mathrm{TPC}}$ is used for continuous closed-loop power adjustments and the eNodeB sends transmit power control (TPC) command to set the value of $\mathrm{f}_{\mathrm{TPC}}$. Using the values of Table I in (7), the transmit power of UE in macrocell and small cell can be shown as

$P u($ macro $)=-82+0.8 \times P L($ macro $)$

And

$P u($ small $)=-100+0.8 \times P L($ small $)$

respectively. The transmit power of UE, as derived from (8) and (9), is plotted against the distance of the UE from the macro eNodeB and the plot is shown in Figure 2. Handover triggering, for both existing and proposed cases, is shown in this figure. The maximum uplink transmit power is found as $15.63 \mathrm{dBm}$ for the existing case while for the proposed case, it is $8.27 \mathrm{dBm}$, which shows a saving of maximum uplink transmit power by $47 \%$. Also, the uplink power is significantly lower within around $300 \mathrm{~m}$ region between the eNodeBs of macrocell and small cell. As Figure 2 shows, this $300 \mathrm{~m}$ region, in the existing scheme, uses the top level of uplink transmit power. Moreover, this $300 \mathrm{~m}$ region starts from a distance of $400 \mathrm{~m}$ from the macro eNodeB, and so, the UE must be close to a few neighboring eNodeBs in the system. Since the UE radiates equally in all horizontal directions, it will cause heavy interference to those eNodeBs. Therefore, the substantial reduction of uplink transmit power, in this same region, as achieved in the proposed scheme, will be greatly helpful in the mitigation of interference in uplink spectrum in HetNets.

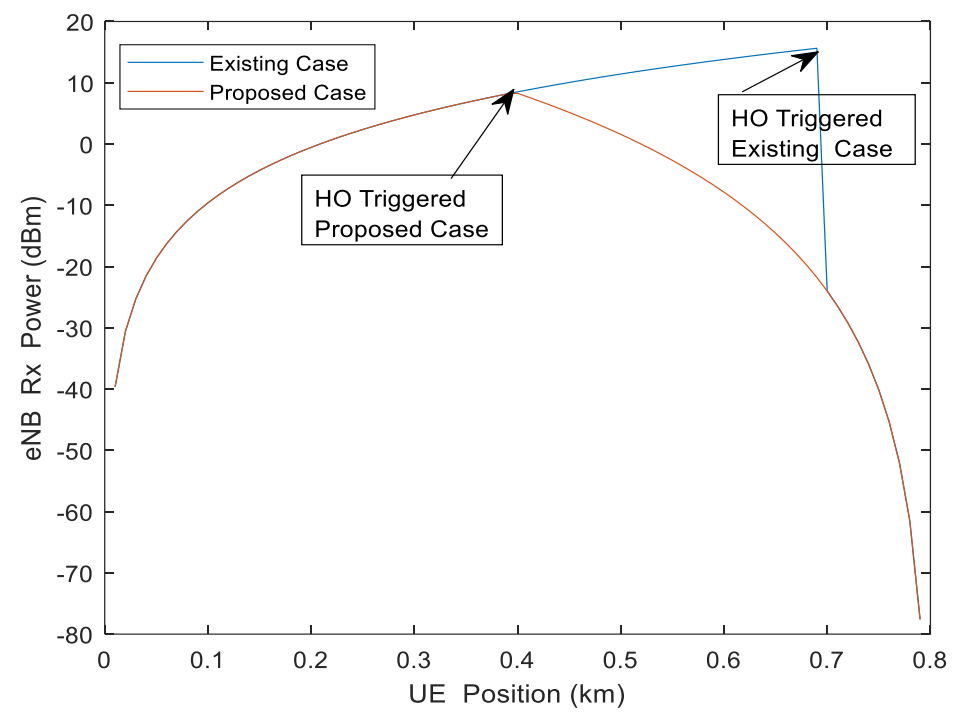

Fig. 2 UE transmit power vs. distance from the macro eNodeB as the UE moves from macrocell to small cell 
Similarly, when the UE moves from small cell to macrocell, the condition to begin sending measurement reports can be shown as

$$
\operatorname{Pr}(\text { macro })-\text { Hysteresis }>\operatorname{Pr}(\text { small })+A 3_{\text {offset }}
$$

For the existing scheme, the simulation results show that the condition of (10) is fulfilled at a distance $d=188.3 \mathrm{~m}$ from the eNodeB of small cell. Using (5) the handover is triggered at $d^{\prime}=190 \mathrm{~m}$. Since $d^{\prime} \approx d$, CSO is set so as to make $d=400 \mathrm{~m}$. To determine this desired $\mathrm{CSO}$ value, $\mathrm{CSO}_{\text {stom }}$, (10) can be modified as

$$
\operatorname{Pr}(\text { small })+\text { A3_offset }+ \text { CSO }_{\text {stom }}=\operatorname{Pr}(\text { macro })-\text { Hysteresis }
$$

which gives the value of CSOstom equal to $29 \mathrm{~dB}$. The transmit power of UE is plotted against the distance of the UE from the eNodeB of small cell and the plot is shown in Figure 3.

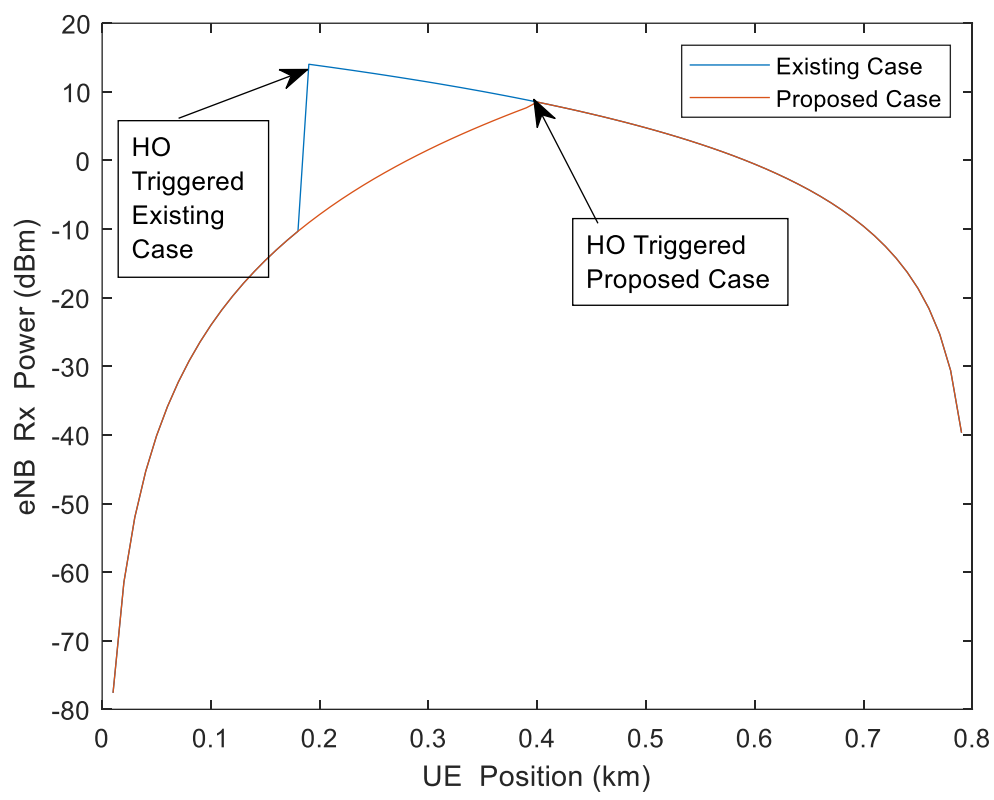

Fig. 3 UE transmit power vs. distance from the eNodeB of small cell as the UE moves from small cell to macrocell

Handover triggering, for both existing and proposed cases, is shown in Figure 3 for UE movement from small cell to macrocell. For the existing case, the maximum uplink transmit power is found as $14.02 \mathrm{dBm}$ while for the proposed case, it is $8.51 \mathrm{dBm}$. Thus, the maximum uplink transmit power is saved by $39.3 \%$. Also, the uplink power is significantly lower within around $210 \mathrm{~m}$ region between the eNodeBs of macrocell and small cell. As Figure 3 shows, this $210 \mathrm{~m}$ region uses the top level of uplink transmit power. This $210 \mathrm{~m}$ region starts from a distance of $190 \mathrm{~m}$ from the eNodeB of small cell, and so, the UE will be pretty close to a few neighboring eNodeBs in the system. The UE, with equal radiation in all horizontal directions, will cause significant interference to those eNodeBs. Therefore, the substantial reduction of uplink transmit power, in this same region, as achieved in the proposed scheme, will be greatly helpful in the mitigation of interference in uplink spectrum in HetNets.

It is thus demonstrated that the proposed scheme, with uplink only data transfer, achieves a significant reduction in the uplink transmit power. In this demonstration, the achievement 
has $7.7 \%$ more saving of maximum uplink transmit power and $90 \mathrm{~m}$ wider region of uplink power saving, when the UE moves from macrocell to small cell, compared to when the UE moves from small cell to macrocell. Section 4 highlights the reasons for this difference in achievements. The reduction in the uplink transmit power mitigates interference greatly and results in other benefits, depicted in Section 4

\section{CONCLUSION}

The HetNets are popular in LTE deployment but it poses additional challenges, especially, due to its complicated interference scenario. Therefore, interference mitigation is an important concern for LTE HetNets. The proposed scheme, by shifting the handover boundary from its conventional location for the case of uplink only data transfer, can substantially mitigate the interference in uplink spectrum. It reduces, for those UEs, the required uplink power transmission within a good part of the cell area, in which the uplink transmit power approaches its maximum in the system. The reduced interference can improve the spectral efficiency by allowing the use of higher MCS and reducing packet retransmissions. The additional benefits, of the proposed scheme, include less draining of UE battery and less clipping off power transmission level by the UE capability. All these benefits are more pronounced when the UE moves from macrocell to small cell compared to the UE movement in the opposite direction. The effectiveness of the proposed method is conspicuous from the simulation results.

\section{REFERENCES}

[1] Mohammad T. Kawser, "LTE Air Interface Protocols", Artech House, Boston, USA (2011). ISBN: 9781-60807-201-9

[2] Mohammad T. Kawser, Mohammad R. Islam, Kazi I. Ahmed, Mir R. Karim, Jaeed B. Saif, "Efficient Resource Allocation and Sectorization for Fractional Frequency Reuse (FFR) in LTE Femtocell Systems", Radioengineering Journal, vol. 24, no. 4, (2015), pp. 940-947. DOI: 10.13164/re.2015.0940

[3] Kerttula et al., "Dynamic TDD in LTE small cells", EURASIP Journal on Wireless Communications and Networking, (2016), 2016:194. DOI: 10.1186/s13638-016-0696-z

[4] Supratim Deb, Pantelis Monogioudis, "Learning Based Uplink Interference Management in 4G LTE Cellular Systems", IEEE/ACM Transactions on Networking, vol. 23, issue. 2, (2015), pp. 398-411.

[5] Alessandro Chiumento, Sofie Pollin, Claude Desset, Liesbet Van der Perre, Rudy Lauwereins, "Scalable LTE interference mitigation solution for HetNet deployment.", IEEE Wireless Communications and Networking Conference Workshops (WCNCW), (6-9 April, 2014), Istanbul, Turkey. DOI: 10.1109/WCNCW.2014.6934859

[6] Xu, W. \& Zhang, H., "Uplink interference mitigation for heterogeneous networks with user-specific resource allocation and power control", J Wireless Com Network (2014). 2014:55. https://doi.org/10.1186/1687-1499-2014-55.

[7] Mohammad T. Kawser, Mohammad R. Islam, Muhammad R. Rahim, Muhammad A. Masud, "Versatile Controllability over Cell Switching for Speedy Users in LTE HetNets", Radioengineering Journal, vol. 26, no.1,(2017), pp.179-190. DOI: 10.13164/re.2017.0179

[8] Aamod Khandekar, Naga Bhushan, Ji Tingfang, Vieri Vanghi, "LTE Advanced: Heterogeneous Networks", In Proceedings of IEEE European Wireless Conference (EW). Lucca, Italy, (12-15 April, 2010), pp. 978-982. DOI: 10.1109/EW.2010.5483516.

[9] 3GPP TS 36.331, "Evolved Universal Terrestrial Radio Access (E-UTRA); Radio Resource Control (RRC)", Release 15, (2018). 